\title{
A New Measure to Evaluate the Consistency of a Set of Decision Rules Extracted From a Decision Table
}

\author{
Nguyen Duc Thuan, Member, IACSIT, Nguyen Xuan Huy
}

\begin{abstract}
Two classical measures, approximation accuracy and consistency degree can be employed to evaluate the decision performance of a decision table. However, these two measures cannot give elaborate depictions of certainty and consistency of a decision table when their values equal to zero. Yuhua Qian et al have introduced three new measures for evaluating the decision performance of a decision-rule set extracted from a decision table, but these measures still have some limitations. In this paper, we propose a new measure and theoretically study to evaluate the consistency of a set of decision rules extracted from a decision table. Experimental analyses on two practical data sets also show our measure will be suited for evaluating the decision performance of a decision-rule than measures of Yuhua Qian et al.
\end{abstract}

Index Terms - accuracy degree, consistency degree, decision table, decision evaluation, rough set theory

\section{INTRODUCTION}

Rough set theory proposed by Pawlak has become a popular mathematical framework for the analysis of a vague description of an object, pattern recognition, image processing, feature selection, conflict analysis, decision support, datamining and knowledge discovery from large data set.

In recent years, how to evaluate the decision performance of a decision rule has become a very important issue in rough set theory. Many authors have proposed measures based on information entropy for this problem. Several other measures such as certainty measure and support measure are often used to evaluate a decision rule. However, all of the above measures are only defined for a single decision rule and are not suitable for measuring the decision performance of a rule set. Two measures: certainty measure and support measure, in some sense, could be regarded as measures for evaluating the decision performance of all decision-rules generated from a complete decision table (Pawlak). Nevertheless, they have some limitations. For instance, the certainty and consistency of a rule set could not be well characterized by the approximation accuracy and consistency degree when their values reach zero. As we know, when the approximation accuracy or consistency degree

Manuscript received April, 14, 2009.

Nguyen Duc Thuan is with the Department of Information Systems, Nha Trang University, Vietnam (phone: +84 58 905444448; fax: +84 58 3831147).

Nguyen Xuan Huy is with the Institute of Information Technology Vietnamese Academy of Science and Technology, Hanoi, Vietnam equal to zero, it is only implied that there is no decision rule with the certainty of one in the complete decision table. To overcome the shortcomings of the existing measures, Yuhua Qian et al have introduced three new measures for evaluating the decision performance of a decision-rule set extracted from a decision table. In their papers, they present properties of these three measures [1,2,3]. Although these measures have many good properties, the consistency measure has limitation: it is not monotony as the same classical measure. In this paper, we propose a new measure to evaluate the consistency of a set of decision rules extracted from a decision table to change the consistency measure of group authors.

The paper is organized as follows. Section 2 briefly introduces information system, decision table, partial relation decision rule, position region reduct. Section 3, presents three measures and properties. In section 4 , our new consistency measure is introduced as well as its use on two data sets. At last, the paper is concluded with a summarization in section 5.

\section{BASIC CONCEPTS}

An information system is a pair $\mathrm{S}=(\mathrm{U}, \mathrm{A})$, where $\mathrm{U}$ is a non-empty, finite set of objects and is called the universe and $\mathrm{A}$ is a non-empty, finite set of attributes. For each $a \in A$, $a: U \rightarrow V_{a}$, where $V_{a}$ is the domain of $a$.

Each non-empty subset $\mathrm{B} \subseteq \mathrm{A}$ determines an indiscernibility relation in the following way:

$$
R_{B}=\{(x, y) \in U \times U \mid a(x)=a(y), \forall a \in B\}
$$

The relation $R_{B}$ partitions $U$ into some equivalence classes given by:

$U / R_{B}=\left\{[\mathrm{x}]_{B} \mid \mathrm{x} \in U\right\}$, where $[\mathrm{x}]_{B}$ denotes the equivalence class determined by $x$ with respect to $B$, i.e.,

$$
[x]_{B}=\left\{y \in U \mid(x, y) \in R_{B}\right\}
$$

A partial relation $\preceq$ on the family $\{\mathrm{U} / \mathrm{B} \mid \mathrm{B} \subseteq \mathrm{A}\}$ is defined as follows:

$U / P \preceq U / Q$ if and only if: $\forall \mathrm{P}_{\mathrm{i}} \in \mathrm{U} / \mathrm{P}$, there exists $\mathrm{Q}_{\mathrm{j}} \in \mathrm{U} / \mathrm{Q}$ such that $\mathrm{P}_{\mathrm{i}} \subseteq \mathrm{Q}_{\mathrm{j}}$. In this case, we say $Q$ is coarser than $P$ or $P$ is finer than $Q$.

A decision table is an information system $\mathrm{S}=(\mathrm{U}, \mathrm{C} \cup \mathrm{D})$, with $C \cap D=\phi), C$ is called a condition attribute set, a element of $\mathrm{D}$ is called a decision attribute, and $\mathrm{D}$ is called a decision attribute set. Suppose $U / C=\left\{X_{1}, X_{2}, . ., X_{m}\right\}$ and U/D 
$=\left\{\mathrm{Y}_{1}, \mathrm{Y}_{2}, . ., \mathrm{Y}_{\mathrm{n}}\right\}$.

A condition class $\mathrm{X}_{\mathrm{i}} \in \mathrm{U} / \mathrm{C}$ is said to be consistent if $\mathrm{d}(x)=$ $\mathrm{d}(y), \forall x, y \in \mathrm{X}_{\mathrm{i}}$ and $\forall \mathrm{d} \in \mathrm{D}$; a decision class $\mathrm{Y}_{\mathrm{j}} \in \mathrm{U} / \mathrm{D}$ is said to be conversely consistent if $\mathrm{a}(x)=\mathrm{a}(y), \forall x, y \in \mathrm{Yj}$ and $\forall a \in \mathrm{C}$.

A decision table $S=(U, C \cup D)$ is said to be consistent if every condition classes $\mathrm{X}_{\mathrm{i}} \in \mathrm{U} / \mathrm{C}$ is consistent. It is easy to see that if U/C $\preceq$ U/D then $S=(U, C \cup D)$ is said to be consistent.

One can define if U/D $\preceq U / C$, then $S$ is said to be conversely consistent.

Let $\mathrm{S}=(\mathrm{U}, \mathrm{C} \cup \mathrm{D})$ be a decision table, $X_{i} \in \mathrm{U} / \mathrm{C}, Y_{j} \in \mathrm{U} / \mathrm{D}$ and $X_{i} \cap Y_{j} \neq \varnothing$. By $\operatorname{des}\left(X_{i}\right)$ and $\operatorname{des}\left(Y_{j}\right)$, we denote the descriptions of the equivalence classes $X_{i}$ and $Y_{j}$ in the decision table $\mathrm{S}$. A decision rule is formally defined as:

$$
Z_{i j}: \operatorname{des}\left(\mathrm{X}_{\mathrm{i}}\right) \rightarrow \operatorname{des}\left(\mathrm{Y}_{\mathrm{j}}\right) \text {. }
$$

Certainty measure and support measure of decision rule $\mathrm{Z}_{\mathrm{ij}}$ are defined as follows:

$$
\mu\left(\mathrm{Z}_{\mathrm{ij}}\right)=\left|\mathrm{X}_{\mathrm{i}} \cap \mathrm{Y}_{\mathrm{j}}\right| /\left|\mathrm{X}_{\mathrm{i}}\right| \text { and } s\left(\mathrm{Z}_{\mathrm{ij}}\right)=\left|\mathrm{X}_{\mathrm{i}} \cap \mathrm{Y}_{\mathrm{j}}\right| / \mathrm{U} \mid
$$

It is clear that $\mu\left(\mathrm{Z}_{\mathrm{ij}}\right)$ and $\mathrm{s}\left(\mathrm{Z}_{\mathrm{ij}}\right)$ of a decision rule $\mathrm{Z}_{\mathrm{ij}}$ falls into the interval $\left[\frac{1}{|U|}, 1\right]$. We also denote $\left|Z_{i j}\right|$ by $\left|X_{i} \cap Y_{j}\right|$.

Pawlak proposed consistency measure of a decision table $S$ is

$$
C_{C}(D)=\frac{\sum_{i=1}^{n}\left|\underline{C} Y_{i}\right|}{|U|}
$$

It is clear that $\mathrm{S}$ is a decision conversely consistent table and consistency table: $\mathrm{C}_{\mathrm{C}}(\mathrm{D})=0$.

$C$-positive region of $D$ is determined:

$$
\operatorname{POS}_{C}(D)=\bigcup_{Y \in U \mid I N D(D)} \underline{C}(Y)=\bigcup_{i=1}^{n} \underline{C}\left(Y_{i}\right)
$$

If $\mathrm{B} \subseteq \mathrm{C}$ satisfies the following conditions:

$$
\begin{aligned}
& \text { 1. } \mathrm{POS}_{\mathrm{C}}(\mathrm{D})=\mathrm{POS}_{\mathrm{B}}(\mathrm{D}) \\
& \text { 2. } \forall \mathrm{a} \in C-B, \operatorname{POS}_{\mathrm{C}}(\mathrm{D})=\operatorname{POS}_{\mathrm{C}-\{\mathrm{a}\}}(\mathrm{D})
\end{aligned}
$$

$B$ is a positive reduct of $D$ with respect to $C$.

\section{DECISION RULE AND DECISION PERFORMANCE MEASUREMENT IN DECISION TABLES}

Yuhua Qian et al have introduced three new measures: certainty measure, consistency measure and support measure for evaluating the decision performance of a decision-rule set extracted from a decision table, they were defined as follows:

Definition 3.1 [1]: Let $\mathrm{S}=(\mathrm{U}, \mathrm{C} \cup \mathrm{D})$ be a decision table, and RULE $=\left\{Z_{i j} \mid Z_{i j}\right.$ : $\left.\operatorname{des}\left(X_{i}\right) \rightarrow \operatorname{des}\left(Y_{j}\right), X_{i} \in \mathrm{U} / \mathrm{C}, Y_{j} \in \mathrm{U} / \mathrm{D}\right\}$, Certainty measure $\alpha$ of $\mathrm{S}$ is defined as:

$$
\alpha(S)=\sum_{i=1}^{m} \sum_{j=1}^{n} s\left(Z_{i j}\right) \mu\left(Z_{i j}\right)=\sum_{i=1}^{m} \sum_{j=1}^{n} \frac{\left|X_{i} \cap Y Y_{j}\right|^{2}}{|U|\left|X_{i}\right|}
$$

Consistency measure $\beta$ of $S$ is defined as:

$$
\beta(S)=\sum_{i=1}^{m} \frac{\left|X_{i}\right|}{|U|}\left[1-\frac{4}{\left|X_{i}\right|} \sum_{j=1}^{N_{i}}\left|X_{i} \cap Y_{j}\right| \mu\left(Z_{i j}\right)\left(1-\mu\left(Z_{i j}\right)\right)\right]
$$

Support measure $\gamma$ of $S$ is defined as:

$$
\gamma(S)=\sum_{i=1}^{m} \sum_{j=1}^{n} s^{2}\left(Z_{i j}\right)=\sum_{i=1}^{m} \sum_{j=1}^{n} \frac{\left.\left|X_{i} \cap Y\right|_{j}\right|^{2}}{|U|^{2}}
$$

Where $\mu\left(Z_{i j}\right)$ and $s\left(Z_{i j}\right)$ are the certainty degree and support degree of the rule $Z_{i j}$ respectively.

\section{NEW MEASURES OF DECISION RULE AND DECISION PERFORMANCE MEASUREMENT IN DECISION TABLES}

In this section, we introduce new measures of decision rule and decision performance measurement in decision tables in rough set theory. Furthermore, we prove some important properties of them. The comparisons of values of measures with the numbers of features in two data sets from UCI Repository of machine learning databases are presented.

Definition 4.1: Let $\mathrm{S}=(\mathrm{U}, \mathrm{C} \cup \mathrm{D})$ be a decision table, and RULE $=\left\{Z_{i j} \mid Z_{i j}: \operatorname{des}\left(X_{i}\right) \rightarrow \operatorname{des}\left(Y_{j}\right), X_{i} \in \mathrm{U} / \mathrm{C}, Y_{j} \in \mathrm{U} / \mathrm{D}\right\}$, Certainty measure $\tau \alpha$ of $S$ is defined as:

$$
\tau \alpha(S)=1-\sum_{i=1}^{m} \sum_{j=1}^{n} \frac{\left|X_{i} \cap Y_{j}\right|}{\left|X_{i}\right|} \frac{\left|X_{i} \cap Y_{j}^{C}\right|}{|U|}
$$

Consistency measure $\tau \beta$ of $S$ is defined as:

$$
\tau \beta(S)=1-\frac{n}{n-1} \sum_{i=1}^{m} \sum_{j=1}^{n} \frac{\left|X_{i} \cap Y_{j}\right|}{\left|X_{i}\right|} \frac{\left|X_{i} \cap Y_{j}^{C}\right|}{|U|}
$$

Where $\mu\left(\mathrm{Z}_{\mathrm{ij}}\right)$ and $s\left(\mathrm{Z}_{\mathrm{ij}}\right)$ are the certainty degree and support degree of the rule $Z_{i j}$ respectively.

Remark 4.1: For support measure, we use support measure of group authors (Yuhua Qian et al.). It means support measure $\gamma$ of $\mathrm{S}$.

Theorem 4.1: Let $\mathrm{S}=(\mathrm{U}, \mathrm{C} \cup \mathrm{D})$ be a decision table, and RULE $=\left\{Z_{i j} \mid Z_{i j}: \operatorname{des}\left(X_{i}\right) \rightarrow \operatorname{des}\left(Y_{j}\right), X_{i} \in \mathrm{U} / \mathrm{C}, Y_{j} \in \mathrm{U} / \mathrm{D}\right\}$, certainty measure $\tau \alpha$ of $S$ is certain measure $\alpha$.

Proof: IT IS EASY TO SEE THAT

$$
\left|X_{i} \cap Y_{j}^{C}\right|=\left|X_{i}\right|-\left|X_{i} \cap Y_{j}\right| .
$$

THEREFORE, WE HAVE

$$
\begin{aligned}
& \tau \alpha(S)=1-\sum_{i=1}^{m} \sum_{j=1}^{n} \frac{\left|X_{i} \cap Y_{j}\right|}{\left|X_{i}\right|} \frac{\left|X_{i} \cap Y_{j}^{C}\right|}{|U|} \\
& =1-\sum_{i=1}^{m} \sum_{j=1}^{n} \frac{\left|X_{i} \cap Y_{j}\right|}{\left|X_{i}\right|}\left(\frac{\left|X_{i}\right|-\left|X_{i} \cap Y_{j}\right|}{|U|}\right) \\
& =1-\sum_{i=1}^{m} \sum_{j=1}^{n}\left(\frac{\left|X_{i} \cap Y_{j}\right|}{|U|}-\frac{\left|X_{i} \cap Y_{j}\right|^{2}}{|U|\left|X_{i}\right|}\right) \\
= & 1-\sum_{i=1}^{m} \sum_{j=1}^{n} \frac{\left|X_{i} \cap Y_{j}\right|}{|U|}+\sum_{i=1}^{m} \sum_{j-1}^{n} \frac{\left|X_{i} \cap Y_{j}\right|^{2}}{\left|X_{i}\right||U|}
\end{aligned}
$$

Since $\sum_{i=1}^{m} \sum_{j=1}^{n} \frac{\left|X_{i} \cap Y_{j}\right|}{|U|}=1$. Therefore, we can get that:

$$
1-\sum_{i=1}^{m} \sum_{j=1}^{n} \frac{\left|X_{i} \cap Y_{j}\right|}{|U|}+\sum_{i=1}^{m} \sum_{j-1}^{n} \frac{\left|X_{i} \cap Y_{j}\right|^{2}}{\left|X_{i}\right||U|}=\sum_{i=1}^{m} \sum_{j-1}^{n} \frac{\left|X_{i} \cap Y_{j}\right|^{2}}{\left|X_{i}\right||U|}
$$

$=\alpha(\mathrm{S})$. THIS COMPLETES THE PROOF. 
Remark 4.2: As we see theorem 4.1, $\tau \alpha$ measure is $\alpha$ measure. So formula of $\tau \alpha$ has a common component with the other measures. It is convenient for programming when we use these measures concurrently.

We can see the important properties of $\tau \alpha$ or $\alpha$ measure in $[1,2,3,4]$. We can list as follows:

Theorem 4.2 [1]: (Extreme) Let $\mathrm{S}=(\mathrm{U}, \mathrm{C} \cup \mathrm{D})$ be a decision table, and RULE $=\left\{Z_{i j} \mid Z_{i j}: \operatorname{des}\left(X_{i}\right) \rightarrow \operatorname{des}\left(Y_{j}\right)\right.$, $\left.X_{i} \in \mathrm{U} / \mathrm{C}, Y_{j} \in \mathrm{U} / \mathrm{D}\right\}$

For any $Z_{i j} \in$ RULE, If $\mu\left(Z_{i j}\right)=1$, then the measure $\tau \alpha(\mathrm{S})$ achieves its maximum value 1 .

If $m=1$ and $n=|U|$, then the measure $\tau \alpha(S)$ achieves its minimum value $\frac{1}{|U|}$.

Theorem 4.3 [1]: Let $S_{1}=\left(U, C_{1} \cup D_{1}\right), S_{2}=\left(U, C_{2} \cup D_{2}\right)$ be two conversely consistent decision tables. If $\mathrm{U} / \mathrm{C}_{1}=\mathrm{U} / \mathrm{C}_{2}$ and $\mathrm{U} / \mathrm{D}_{2} \preceq \mathrm{U} / \mathrm{D}_{1}$ then $\tau \alpha\left(\mathrm{S}_{1}\right) \geq \tau \alpha\left(\mathrm{S}_{2}\right)$.

Theorem 4.4 [4]: Let $S=(U, C \cup D), S^{\prime}=(U, B \cup D)$ be two decision tables, where $S$ is consistent, $B \subseteq C$. If $B$ is a positive-region reduct of $\mathrm{C}$, then

$$
\tau \alpha(S)=\tau \alpha\left(S^{\prime}\right)
$$

Theorem 4.5 [4]: Let $S=(U, C \cup D), S^{\prime}=(U, B \cup D)$ be two decision tables, $\mathrm{B} \subseteq \mathrm{C}$. If $\mathrm{B}$ is a positive-region reduct of $\mathrm{C}$, then

$$
\tau \alpha(S) \geq \tau \alpha\left(S^{\prime}\right)
$$

Now, we introduce measure $\tau \beta$ and some results which have been found by us:

Theorem 4.6: (Extreme) Let $\mathrm{S}=(\mathrm{U}, \mathrm{C} \cup \mathrm{D})$ be a decision table, and $\operatorname{RULE}=\left\{Z_{i j} \mid Z_{i j}: \operatorname{des}\left(X_{i}\right) \rightarrow \operatorname{des}\left(Y_{j}\right), X_{i} \in \mathrm{U} / \mathrm{C}\right.$, $\left.Y_{j} \in \mathrm{U} / \mathrm{D}\right\}$

1. For every $Z_{i j} \in$ RULE, If $\mu\left(Z_{i j}\right)=1$, then the measure $\tau \beta(S)$ achieves its maximum value 1 .

2. If $m=1$ and $n=|U|$, the measure $\tau \beta(S)$ achieves its minimum value 0 .

Proof: (1) From $\mu\left(Z_{i j}\right)=1 \Leftrightarrow$

$$
\begin{gathered}
\frac{\left|X_{i} \cap Y_{j}\right|}{\left|X_{i}\right|}=1 \Leftrightarrow \frac{\left|X_{i} \cap Y_{j}^{C}\right|}{|U|}=0 \\
\Rightarrow \frac{\left|X_{i} \cap Y_{j}\right|}{|U|} \frac{\left|X_{i} \cap Y_{j}^{C}\right|}{|U|}=0, \forall \mathrm{i}, \mathrm{j} \\
\Rightarrow \tau \beta(S)=1-\frac{n}{n-1} \sum_{i=1}^{m} \sum_{j=1}^{n} \frac{\left|X_{i} \cap Y_{j}\right|\left|X_{i} \cap Y_{j}^{C}\right|}{\left|X_{i}\right|}=1
\end{gathered}
$$

(2) By definition $\tau \beta(\mathrm{S})$, we can write it as:

$$
\begin{aligned}
& \tau \beta(S)=1-\frac{n}{n-1} \sum_{i=1}^{m} \sum_{j=1}^{n} \frac{\left|X_{i} \cap Y_{j}\right|\left|X_{i} \cap Y_{j}^{C}\right|}{\left|X_{i}\right|} \frac{|U|}{\mid} \\
& =1-\frac{n}{n-1}(1-\tau \alpha(S))=1+(\tau \alpha(S)-1) \frac{n}{n-1}
\end{aligned}
$$

IF M $=1$ AND N $=|U|$, THEN THE MEASURE $\tau \alpha(S)$ ACHIEVES ITS
Minimum VAlUe $\frac{1}{|U|}=\frac{1}{n}$ (THEOREM 4.2). Hence, THE MEASURE $\tau \beta(S)$ achieves its minimum value 0 .

$$
\tau \beta(S)_{\min }=1+\left(\frac{1}{n}-1\right)\left(\frac{n}{n-1}\right)=0
$$

This completes the proof.

The monotony of the measure $\tau \beta$ on the conversely consistent decision tables can be found in the following theorems:

Theorem 4.7: Let $S_{1}=\left(U, C_{1} \cup D_{1}\right), S_{2}=\left(U, C_{2} \cup D_{2}\right)$ be two conversely consistent decision tables. If $\mathrm{U} / \mathrm{C}_{1}=\mathrm{U} / \mathrm{C}_{2}$ and $\mathrm{U} / \mathrm{D}_{2} \preceq \mathrm{U} / \mathrm{D}_{1}$ then $\tau \beta\left(S_{1}\right) \geq \tau \beta\left(S_{2}\right)$.

Proof: From Definition 4.1 and theorem 4.3. The monotony of the measure $\tau \beta$ and $\tau \alpha$ are the same. We also directly prove it:

Since $\mathrm{U} / \mathrm{C}_{1}=\mathrm{U} / \mathrm{C}_{2}, \mathrm{~S}_{1}$ and $\mathrm{S}_{2}$ be two conversely consistent decision tables. Suppose:

$$
\begin{aligned}
& \mathrm{U} / \mathrm{C}_{1}=\mathrm{U} / \mathrm{C}_{2}=\left\{\mathrm{X}_{1}, \mathrm{X}_{2}, . ., \mathrm{X}_{\mathrm{m}}\right\} \\
& \mathrm{U} / \mathrm{D}_{1}=\left\{\mathrm{Y}_{1}, \mathrm{Y}_{2}, . ., \mathrm{Y}_{\mathrm{n}}\right\}
\end{aligned}
$$

If $\mathrm{U} / \mathrm{D}_{2} \preceq \mathrm{U} / \mathrm{D}_{1}$, then $\mathrm{U} / \mathrm{D}_{2}=\bigcup_{j=1}^{n}\left\{\bigcup_{k=1}^{t} Y_{j}^{k}\right\}$, where

$$
\mathrm{Y}_{\mathrm{j}}={\underset{k=t}{t_{j-1}+1}}_{j} Y_{j}^{k} \text {, let } \mathrm{t}_{0}=0, \mathrm{t}_{\mathrm{n}}=\mathrm{s}, \mathrm{Z}_{\mathrm{k}}=Y_{j}^{k}
$$

It follows that $U / D_{2}=\left\{Z_{1}, Z_{2}, . ., Z_{s}\right\}$.

We can get that:

$$
\begin{aligned}
& \left|X_{i} \cap Y_{j}\right|\left|X_{i} \cap Y_{j}^{C}\right| \\
& =\left|X_{i} \cap\left(\underset{k=t}{U_{j-1}^{t}} Y_{j}^{k}\right)\right|\left|X_{i} \cap\left(\bigcup_{k=t}^{t_{j-1}^{j}} Y_{j}^{k}\right)^{C}\right| \\
& \leq\left|\bigcup_{k=t_{j-1}^{+1}}^{t_{j}}\left(X_{i} \cap Y_{j}^{k}\right)\right| \mid \bigcup_{k=t}^{t_{j-1}+1}\left(X_{i} \cap\left(Y_{j}^{k}\right) \mid\right. \\
& =\sum_{k=t_{j-1}+1}^{t_{j}}\left|X_{i} \cap Y_{j}^{k}\right|\left|X_{i} \cap\left(Y_{j}^{k}\right)^{C}\right|
\end{aligned}
$$

So, we know that if

$$
\sum_{i=1}^{m} \sum_{j=1}^{n} \frac{\left|X_{i} \cap Y_{j}\right|}{\left|X_{i}\right|} \frac{\left|X_{i} \cap Y_{j}^{C}\right|}{|U|} \leq \sum_{i=1}^{m} \sum_{j=1}^{n} \sum_{k=1}^{s} \frac{\left|X_{i} \cap Y_{j}^{k}\right|}{\left|X_{i}\right|} \frac{\left|X_{i} \cap\left(Y_{j}^{k}\right)^{C}\right|}{|U|}
$$

THEN

$$
\begin{aligned}
& 1-\frac{n}{n-1} \sum_{i-1}^{m} \sum_{j=1}^{n} \frac{\left|X_{i} \cap Y_{j}\right|}{|U|} \frac{\left|X_{i} \cap Y_{j}^{C}\right|}{\left|X_{i}\right|} \\
& \geq 1-\frac{n}{n-1} \sum_{i=1}^{m} \sum_{k=1}^{s} \frac{\left|X_{i} \cap Z_{k}\right|}{|U|} \frac{\left|X_{i} \cap Z_{k}^{C}\right|}{\left|X_{i}\right|}
\end{aligned}
$$

In other words, we have $\tau \beta\left(S_{1}\right) \geq \tau \beta\left(S_{2}\right)$. This completes the proof.

This theorem states that the consistency measure $\tau \beta$ of a conversely consistent decision table increases with its decision classes becoming finer with all $\mu(Z i j)$.

Lemma 4.1: Let $S=(U, C \cup D)$ be a decision table, and 2 
non-empty sets $\mathrm{X}, \mathrm{Y} \subseteq \mathrm{U}$. Suppose $\mathrm{X}=\bigcup_{j=1}^{k} X_{j}, \mathrm{X}_{\mathrm{p}} \cap \mathrm{X}_{\mathrm{q}}=\varnothing$ for any $p \neq q$, i.e. $\left\{X_{1}, X_{2}, . ., X_{k}\right\}$ is a partition of $X$, then

$$
\frac{|X \cap Y|}{|U|} \frac{|X \cap Y C|}{|X|} \geq \sum_{j=1}^{k} \frac{\left|X_{j} \cap Y\right|\left|X_{j} \cap Y C\right|}{|U|} \frac{|X|}{|X|}
$$

And, if $\left|X_{p} \cap Y\right|\left|X_{q} \cap Y C\right|=0$, for any $\mathrm{p} \neq \mathrm{q}$ and $\mathrm{p}, \mathrm{q}=1$,

$2, . ., \mathrm{k}$, we have " $="$.

Proof: Similar to idea of Lemma 4.3 in [5], we can prove it as follows:

Since, $X_{p} \cap X_{q}=\varnothing$ for any $p \neq q$, we have

$$
\begin{gathered}
\frac{|X \cap Y||X \cap Y C|}{|U|} \frac{\left|\bigcup_{j=1}^{k} X_{i} \cap Y\right|}{|X|}=\frac{\left|\bigcup_{j=1}^{k} X_{i} \cap Y^{C}\right|}{|X|} \\
=\sum_{j=1}^{k} \frac{|X j \cap Y|}{|U|} \sum_{j=1}^{k} \frac{\left|X_{j} \cap Y^{C}\right|}{|X|}=\sum_{j=1}^{k} \sum_{p=1}^{k} \frac{\left|X_{j} \cap Y\right|}{|U|} \frac{\left|X_{p} \cap Y^{C}\right|}{|X|} \\
\geq \sum_{j=1}^{k} \frac{\left|X_{j} \cap Y\right|\left|X_{j} \cap Y\right|}{|U|} \frac{|X|}{|X|}
\end{gathered}
$$

It is clearly, if $\left|X_{p} \cap Y\right|\left|X_{q} \cap Y C\right|=0$, for any $\mathrm{p} \neq \mathrm{q}$ and $\mathrm{p}, \mathrm{q}$ $=1,2, . ., \mathrm{k}$, we have "=". This completes the proof.

Theorem 4.8: Let $S_{1}=\left(U, C_{1} \cup D_{1}\right), S_{2}=\left(U, C_{2} \cup D_{2}\right)$ be two conversely consistent decision tables. If $\mathrm{U} / \mathrm{D}_{1}=\mathrm{U} / \mathrm{D}_{2}$ and $\mathrm{U} / \mathrm{C}_{2} \preceq \mathrm{U} / \mathrm{C}_{1}$ then $\tau \beta\left(S_{1}\right) \leq \tau \beta\left(S_{2}\right)$.

Proof: Suppose $U / D_{2}=\left\{Y_{1}, Y_{2}, . ., Y_{n}\right\}, U / C_{1}=\left\{X_{1}, X_{2}, .\right.$. , $\left.\mathrm{X}_{\mathrm{m}}\right\}$,

$$
\text { If } \mathrm{X}_{\mathrm{i}}=\bigcup_{k=t_{i-1}+1}^{t_{i}} X_{i}^{k} \Rightarrow \mathrm{U} / \mathrm{C}_{2}=\bigcup_{i=1}^{m}\left\{\bigcup_{k=t_{i}+1}^{t_{i}} X_{i}^{k}\right\} \text {, let } \mathrm{t}_{0}=0 \text {, }
$$

From lemma 4.1 , for each $\mathrm{j}=1, . ., \mathrm{n}$ and $\mathrm{i}=1, \ldots, \mathrm{m}$, we have:

$$
\frac{\left|X_{i} \cap Y_{j}\right|\left|X_{i} \cap Y_{j}^{C}\right|}{|U|} \geq \sum_{k=t_{i-1}+1}^{t} \frac{\left|X_{i}^{k} \cap Y_{j}\right|}{|U|} \frac{\left|X_{i}^{k} \cap Y_{j}\right|}{\left|X_{i}\right|},
$$

let $\mathrm{t}_{0}=0$. Thus,

$$
\begin{gathered}
\sum_{i=1}^{m} \sum_{j=1}^{n} \frac{\left|X_{i} \cap Y_{j}\right|}{|U|} \frac{\left|X_{i} \cap Y_{j}^{C}\right|}{\left|X_{i}\right|} \\
\geq \sum_{i=1}^{m} \sum_{j=1}^{n} \sum_{k=t_{i=1}}^{t_{i}} \frac{\left|X_{i}^{k} \cap Y_{j}\right|}{|U|} \frac{\left|X_{i}^{k} \cap Y_{j}^{C}\right|}{\left|X_{i}\right|}
\end{gathered}
$$

Then, we can get that

$$
\begin{gathered}
1-\frac{n}{n-1} \sum_{i=1}^{m} \sum_{j=1}^{n} \frac{\left|X_{i} \cap Y_{j}\right| \frac{\left|X_{i} \cap Y_{j}^{C}\right|}{|U|} \frac{X_{i} \mid}{\left|X_{i}^{k} \cap Y_{j}\right|\left|X_{i}^{k} \cap Y_{j}^{C}\right|}}{|U|} . \\
\leq 1-\frac{n}{n-1} \sum_{i=1}^{m} \sum_{j=1}^{n} \sum_{k=t_{i=1}}{ }_{i}{ }_{i} \frac{|U|}{\mid X_{i}}
\end{gathered}
$$

Therefore, we have $\tau \beta\left(S_{1}\right) \leq \tau \beta\left(S_{2}\right)$. This completes the proof.

Lemma 4.2: Let $S_{1}=\left(U, C \cup D_{1}\right), S_{2}=\left(U, B \cup D_{2}\right)$ be two decision tables, if $\mathrm{C} \subseteq \mathrm{B}$ then $\mathrm{U} / \mathrm{B} \preceq \mathrm{U} / \mathrm{C}$ then $\tau \beta\left(S_{2}\right) \leq \tau \beta\left(S_{1}\right)$.
And, $\tau \beta\left(S_{2}\right)=\tau \beta\left(S_{1}\right)$ if $S_{1}, S_{2}$ are consistent decision tables.

Proof: We only consider the case $\tau \beta\left(S_{2}\right)=\tau \beta\left(S_{1}\right)(*)$.

when $\mathrm{U} / \mathrm{B} \preceq \mathrm{U} / \mathrm{C}$ we have $\forall \mathrm{X}_{\mathrm{i}} \in \mathrm{U} / \mathrm{C}, \mathrm{X}_{\mathrm{i}}$ can write $\mathrm{X}_{\mathrm{i}}$ $=\bigcup_{k=1}^{t_{i}} X_{i}^{k}$, where $X_{i}^{k} \in \mathrm{U} / \mathrm{B}$. From lemma 4.1 if $\left|X_{i}^{k} \cap Y_{j}\right|\left|X_{i}^{l} \cap Y_{j}^{C}\right|=0, \forall \mathrm{i}, \mathrm{j}, \mathrm{k} \neq \mathrm{l}$ we have $\tau \beta\left(S_{2}\right)=\tau \beta\left(S_{1}\right)$. So $\left|X_{i}^{k} \cap Y_{j}\right|\left|X_{i}^{l} \cap Y_{j}^{C}\right|=0$ if $\left|X_{i}^{k} \cap Y_{j}\right|\left|X_{i}^{l} \cap Y_{j}^{C}\right|=0$ (1), or $\left|X_{i}^{l} \cap Y_{j}\right|\left|X_{i}^{k} \cap Y_{j}^{C}\right|=0$ (2).

If we have (1), then $\left|X_{i}^{k} \cap Y_{j}\right|=0$ or $\left|X_{i}^{l} \cap Y_{j}^{C}\right|=0$, or $\left|X_{i}^{k} \cap Y_{j}\right|=0,\left|X_{i}^{l} \cap Y_{j}^{C}\right|=0$.

$$
\text { If }\left|X_{i}^{k} \cap Y_{j}\right|=0 \Rightarrow\left|X_{i}^{k} \cap Y_{j}^{C}\right| \neq 0 \Rightarrow\left|X_{i}^{l} \cap Y_{j}\right|=0 \text {. }
$$

Since $X_{i}^{k}, X_{i}^{l}$ are the same, we can say that, $\tau \beta\left(S_{2}\right)=\tau \beta\left(S_{1}\right)$ holds, when $\exists \mathrm{j}_{0}, \forall \mathrm{k}=1$..t $\mathrm{t}_{\mathrm{i}}, X_{i}^{k} \subseteq Y_{j}$. In other words, $\mathrm{S}, \mathrm{S}$ ' are consistent decision tables. In this case, we also have $\mu\left(Z_{\mathrm{ij}}\right)=\mu\left(Z_{\mathrm{ij}}^{k}\right), \forall \mathrm{j}, \mathrm{k}$, where $\mathrm{X}_{\mathrm{i}}=\bigcup_{k=1}^{t_{i}} X_{i}^{k}$.

Theorem 4.9: Let $S_{1}=\left(U, C \cup D_{1}\right), S_{2}=\left(U, B \cup D_{2}\right)$ be two decision tables, if $\mathrm{S}$ is consistent decision table and $\mathrm{B} \subseteq \mathrm{C}$. If $\mathrm{B}$ is a positive reduct of $\mathrm{D}$ with respect to $\mathrm{C}$, then

$$
\tau \beta\left(S_{2}\right)=\tau \beta\left(S_{1}\right)
$$

Proof: It is straightforward from definition positive reduct and lemma 4.2

From lemma 4.2, we also have theorem:

Theorem 4.10: Let $S_{1}=\left(U, C \cup D_{1}\right), S_{2}=\left(U, B \cup D_{2}\right)$ be two decision tables, if $\mathrm{B} \subseteq \mathrm{C}$ and $\mathrm{B}$ is a positive reduct of $\mathrm{D}$ with respect to $\mathrm{C}$, then

\section{$\tau \beta\left(\mathrm{S}_{2}\right) \geq \tau \beta\left(\mathrm{S}_{1}\right)$}

For general decision tables, to illustrate the differences between the consistency measure $\tau \beta$ and the consistency measures: $\beta$ and $\mathrm{C}_{\mathrm{C}}(D)$, we have downloaded two data sets from UCI Repository of machine learning databases [6], which described in table 1 . All condition attributes and decision attributes in two data sets are discrete.

Here, we compare the consistency measure $\tau \beta$ with consistency measures: $\beta$ and $\mathrm{C}_{\mathrm{C}}(D)$ on 2 data sets: Tic-tac-toe, Dermatology. The comparisons of values of three measures with the numbers of features in these 2 data sets are shown in Tables 2-3 and figs 1-2.

TABLE 1 DATA SETS DESCRIPTION.

\begin{tabular}{|c|c|c|c|}
\hline Data sets & Samples & Condition features & $\begin{array}{c}\text { Decision } \\
\text { classes }\end{array}$ \\
\hline Tic-tac-toe & 958 & 9 & 2 \\
\hline Dermatology & 366 & 33 & 6 \\
\hline
\end{tabular}

TABLE $2 c_{c}(D), \beta$ AND $\tau \beta$ WITH DIFFERENT NUMBERS OF FEATURES IN THE DATA SET TIC-TAC-TOE; 


\begin{tabular}{|l|l|l|l|}
\hline \multicolumn{1}{|r|}{ Measures } & $c_{c}(D)$ & $\beta$ & $\tau \beta$ \\
\hline 1 & 0.0000 & 0.1114 & 0.1114 \\
\hline 2 & 0.0000 & 0.1322 & 0.1322 \\
\hline 3 & 0.1253 & 0.2827 & 0.2827 \\
\hline 4 & 0.1628 & 0.3300 & 0.3300 \\
\hline 5 & 0.4186 & 0.5832 & 0.5832 \\
\hline 6 & 0.7766 & 0.8000 & 0.8000 \\
\hline 7 & 0.9436 & 0.9436 & 0.9436 \\
\hline 8 & 1.0000 & 1.0000 & 1.0000 \\
\hline 9 & 1.0000 & 1.0000 & 1.0000 \\
\hline
\end{tabular}

TABLE $3 \quad c_{c}(D), \beta$ AND $\tau \beta$ WITH DIFFERENT NUMBERS OF FEATURES IN THE DATA SET DERMATOLOGY

\begin{tabular}{|l|l|l|l|}
\hline Features & $c_{c}(D)$ & $\beta$ & $\tau \beta$ \\
\hline 1 & & $\beta$ & \\
\hline 2 & 0.0000 & 0.3350 & 0.0854 \\
\hline 3 & 0.0109 & 0.3164 & 0.1581 \\
\hline 6 & 0.0437 & 0.2821 & 0.2960 \\
\hline
\end{tabular}

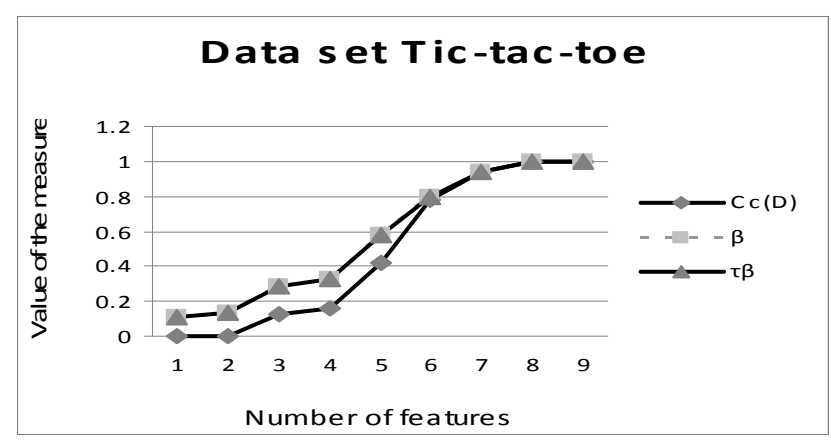

Figure 1. Variation of the consistency measures: $\tau \beta, \beta$ and consistency degree with the number of features (data set

Tic-Tac-Toe)

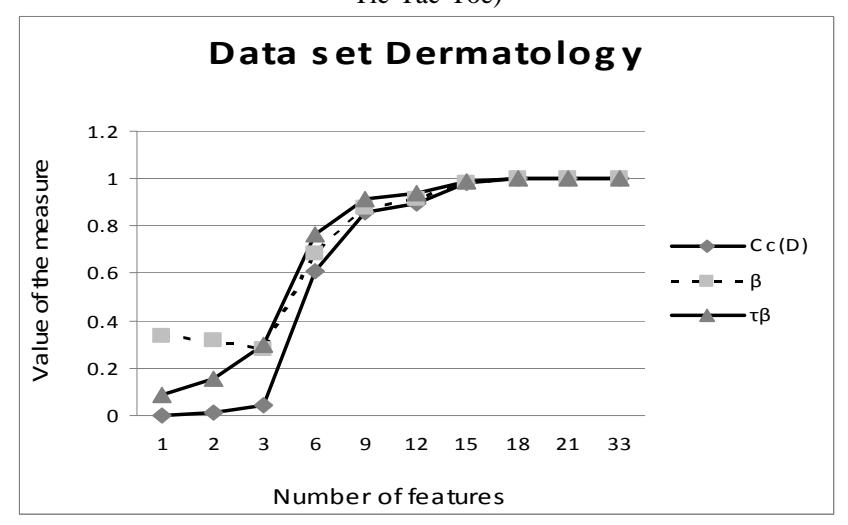

Figure 2. Variation of the consistency measures: $\tau \beta, \beta$ and consistency degree with the number of features (data set Dermatology)

It can be seen from tables 2-3 that the value of consistency measure $\tau \beta$ is biggest for the same number of selected features. However, $\tau \beta$ and $\mathrm{C}_{\mathrm{C}}(D)$ are the same monotony on all number of selected features, but $\beta$ is not, it increases with its decision classes becoming finer when $\forall \mu\left(Z_{i j}\right) \leq 1 / 2$, and decreases with its decision class becoming finer when $\forall \mu\left(Z_{i j}\right) \geq 1 / 2$ and it decreases with its condition classes becoming finer when $\forall \mu\left(Z_{i j}\right) \leq 1 / 2$, and increases with its decision class becoming finer when $\forall \mu\left(Z_{i j}\right) \geq 1 / 2$ (We also can see this through theorem 6 and theorem 7 in [1]).

\section{CONCLUSION}

In this paper, we introduce two measures for evaluating a decision rule or decision table such as certainty measure and consistency measure. Note: certainty measure by Yuahua Qian et al is identical to the certainty measure of mine. We have proposed our measures to overcome the limitations of previous measures. Our measures have simple formulas, and they have common components. So, they are convenient for programming when we use these measures concurrently. We have proved theorems and properties of our measures. The experimental analyses on the 2 practical decision tables show that these new measures are adequate for evaluating the decision performance of a decision-rule set extracted from a decision table in rough set theory.

\section{REFERENCES}

[1] Yuhua Qian, Jiye Liang, Deyeu Li, Haiyun Zhang, Chuangyin Dang, Measures of evaluating the decision performace of a decision table in rough set theory, Information Sciences 178(2008) $181-202$.

[2] Yuhua Qian, Jiye Liang, Chuangyin Dang, Converse approximation and rule extraction from decision tables in rough set theory, Computers and Mathematics with Applications 55(2008) 1754-1765.

[3] Yuhua Qian, Chuangyin Dang, Jiye Liang, Haiyun Zhang, Jianmin Ma, On the valuation of the decision performance of an incomplete decision table, Data \& Knowledge Engineering 65(2008) 373-400.

[4] Wei Wei, Jiye Liang, Yuhua Qian, Change Mechanism of a Decision Table's Decision Performance Caused by Attribute Reductions, Granular Computing, 2008, GrC 2008, IEEE Internation Conference p $638-643$.

[5] Nguyen Thanh Tung, Nong Thi Hoa, A new method construction of decision tree based on rough set theory, Proceedings of National Conference, Hue 12-13, 2008, 229-241.

[6] The UCI machine learning repository, <http://mlearn.ics.uci.edu/MLRepository.html >.

[7] Z. Pawlak, Rough sets, Theoretical Aspects of Reasoning about Data, Kluwer Academic Publishers, London 1991.

First Author Name: Nguyen Duc Thuan was born in Hue, Vietnam, 1962 Brief Biographical History:

-1985, Bachelor in Mathematics- Lecturer of Hue University, Vietnam.

-1998, Master in Information Technology, Lecturer of Nha Trang University, Vietnam.

-2006, Ph.D Student in Institute of Information Technology, Vietnamese academy of Science and Technology.

Current research: Rough set, Datamining, Distributed Database...

Second Author name Nguyen Xuan Huy was born in Hai Duong, Vietnam, 1944.

Brief Biographical History:

1973, Bachelor in Mathematics of Leningrad (Russia)

1982, 1990, Doctor and Post Doc in Information Technology of

Russian Academy of Science and Technology

- At present, Associate Professor of Vietnamese Academy of Science and Technology 